\title{
RF MEMS Sequentially Reconfigurable Sierpinski Antenna on a Flexible Organic Substrate With Novel DC-Biasing Technique
}

\author{
Nickolas Kingsley, Member, IEEE, Dimitrios E. Anagnostou, Member, IEEE, \\ Manos Tentzeris, Senior Member, IEEE, and John Papapolymerou, Senior Member, IEEE
}

\begin{abstract}
Devices and systems that use RF microelectromechanical systems (RF MEMS) switching elements typically use one switch topology. The switch is designed to meet all of the performance criteria. However, this can be limiting for highly dynamic applications that require a great deal of reconfigurability. In this paper, three sets of RF MEMS switches with different actuation voltages are used to sequentially activate and deactivate parts of a multiband Sierpinski fractal antenna. The implementation of such a concept allows for direct actuation of the electrostatic MEMS switches through the RF signal feed, therefore eliminating the need for individual switch dc bias lines. This reconfigurable antenna was fabricated on liquid crystal polymer substrate and operates at several different frequencies between 2.4 and $18 \mathrm{GHz}$ while maintaining its radiation characteristics. It is the first integrated RF MEMS reconfigurable antenna on a flexible organic polymer substrate for multiband antenna applications. Simulation and measurement results are presented in this paper to validate the proposed concept.

[2007-0013]
\end{abstract}

Index Terms-Liquid crystal polymer (LCP), multiband, reconfigurable antenna, $\mathrm{RF}$ microelectromechanical systems (RF MEMS), Sierpinski fractal antenna.

\section{INTRODUCTION}

$\mathbf{T}$ HE RF microelectromechanical systems (RF MEMS) switches are quickly becoming a popular switching element among microwave designers. Their low loss, small size, excellent isolation, and low distortion make them attractive for a wide range of applications. They have already been integrated into filters [1], [2], antennas [3]-[5], phase shifters [6], and many other RF devices.

Designers typically optimize the MEMS geometry to meet a given specification. Switches can be made wide and short or narrow and long to meet a specific size requirement. The materials can be tailored to meet a desired actuation voltage.

Manuscript received January 22, 2007; revised April 27, 2007. This work was supported by the National Science Foundation (NSF) under Grant ECS0500860. Subject Editor S. Lucyszyn.

N. Kingsley was with the School of Electrical and Computer Engineering, Georgia Institute of Technology, Atlanta, GA 30308 USA. He is now with the Modeling and Design Group, Auriga Measurement Systems, Lowell, MA 01854 USA (e-mail: kingsley@gatech.edu).

D. E. Anagnostou is with the Electrical and Computer Engineering Department, South Dakota School of Mines and Technology, Rapid City, SD 57701 USA.

M. Tentzeris and J. Papapolymerou are with the School of Electrical and Computer Engineering, Georgia Institute of Technology, Atlanta, GA 30308 USA.

Color versions of one or more of the figures in this paper are available online at http://ieeexplore.ieee.org.

Digital Object Identifier 10.1109/JMEMS.2007.902462
The height of the membrane can be tuned to give a certain level of isolation when in the "OFF" position. The inductive and capacitive regions can be designed to work best at a given frequency. For each application, and in all existing literature, there is typically one switch membrane geometry, and it is used exclusively throughout the system.

However, using only one switch membrane geometry can be limiting if the device needs maximum reconfigurability. It could be beneficial to utilize multiple switch membrane geometries to add an additional level of reconfigurability. For example, to provide the lowest possible loss from the switching element, several different switches could be used in parallel which are tuned for different operating frequencies. As the frequency is changed, the switch that works best at that frequency is used. Since MEMS switches offer excellent "OFF"-state isolation (usually better than $30 \mathrm{~dB}$ ), the presence of the additional switches would have a negligible effect on the device. This same technique could be used to select switches of different impedances, switching speed, isolation, capacitance, etc. A system could also select between ohmic and capacitive switches to operate from dc to very high frequencies. This technique is ideal for applications that need maximum reconfigurability and can tolerate the slight size increase from the additional switches.

To demonstrate the effectiveness of using multiple switch geometries in a working system, this paper presents a threeiteration coplanar waveguide (CPW)-fed Sierpinski gasket monopole antenna that is reconfigured by turning on various RF MEMS switches. Different areas of the antenna geometry are sequentially activated and deactivated by changing the dc voltage present at the RF feed. This method eliminates the need for dc bias lines at each MEMS switch, which will improve the radiation characteristics of the antenna.

In the past decade, fractal or prefractal shapes have been introduced in antenna and array designs. Several of these designs have been extensively studied, including Koch [7]-[9], Hilbert, Peano, Minkowski, and Sierpinski [10], [11] geometrical shapes or array arrangements [10], demonstrating both compactness and multiband behavior. A comprehensive review [12] describes in detail, among others, the multiband function of the Sierpinski gasket monopole and dipole antennas.

In the majority of the published literature, integration has been accomplished on rigid and nonflexible semiconducting or organic polymer substrates such as silicon and FR-4. The idea of integrating RF MEMS switches into a multiband self-similar antenna was first implemented in [3], where the entire system, 


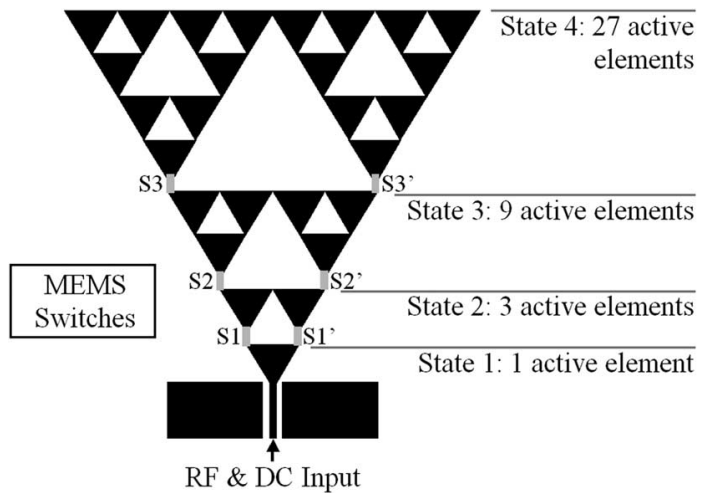

Fig. 1. Illustration of a MEMS reconfigurable Sierpinski antenna. The centerline of the CPW feed provides the RF input and dc voltage for MEMS switch actuation. Switches S1 and S1' actuate at a low voltage, switches S2 and S2' actuate at a medium voltage, and switches S3 and S3' actuate at a high voltage.
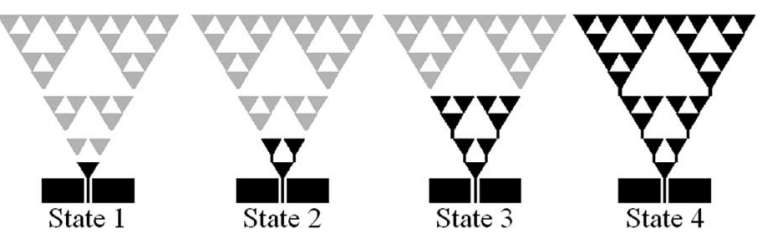

Fig. 2. Four different reconfigurable antenna states: State 1 has no voltage applied, state 2 has a low voltage applied, state 3 has a medium voltage applied, and state 4 has a high voltage applied. The activated (radiating) part of the antenna is darkened.

including the RF MEMS, the planar self-similar antenna, and the CPW-coplanar strip transition, was fabricated on silicon. In this paper, the integration is achieved on a very thin and flexible liquid crystal polymer (LCP) substrate.

Since all of the switches share a common dc feed, this technique provides reconfigurability without the need for additional bias lines. This is advantageous since dc bias lines take up space, add loss, and reduce the bandwidth of a device. This technology is particularly useful for antennas where bias lines can have a pronounced effect on radiation patterns. In this paper, simulation and measurement results are presented with good agreement.

\section{IMPLEMENTATION OF RECONFIGURABILITY}

The implementation of a sequentially activated antenna is shown in Fig. 1. All of the MEMS switches used are singlesupported (cantilever type) and ohmic. Regardless of the applied voltage, the triangular element that is closest to the RF/dc input is always active (Fig. 2, state 1). When no dc voltage is applied, the antenna radiates at its highest frequency.

When a low dc voltage is applied to the signal line, the first set of MEMS switches (S1 and S1') actuate, and this activates the second level of triangular elements (Fig. 2, state 2). The antenna now radiates at a lower frequency. Since all of the switches are ohmic, the low voltage is also present at the membrane of the next set of switches (S2 and S2'). However, these switches are designed to actuate at a higher voltage so they are unaffected by the voltage present.

When a higher dc voltage is applied, the first set of MEMS switches ( $\mathrm{S} 1$ and $\mathrm{S} 1$ ') remains in the "ON" position while the second set of switches (S2 and S2') actuates (Fig. 2, state 3).
This activates the next iteration, consisting of six additional radiating elements. Again, this higher voltage is present at the next set of switch membranes (S3 and S3'), but the electrostatic force created is not sufficient for actuation.

Finally, when the voltage is increased to its highest value, the first two sets of switches (S1 and S1' and S2 and S2') remain in the "ON" position while the remaining set of the switches (S3 and S3') actuates (Fig. 2, state 4). In a way, the voltage cascades from one state to the next like a sequence of overflowing buckets. This technique could not be used if the switches were capacitive since they do not pass dc voltage. The four different states are illustrated in Fig. 2, where all of the activated regions for different voltages are dark in color.

This biasing technique allows for direct actuation of the electrostatic MEMS switches through the RF feed structure. Since only the RF feed is dc grounded, the switches actuate with the use of a floating ground. That is, the signal pin of the CPW feed is connected to the dc cathode, and the ground pins are corrected to the anode of a bias tee. The electrostatic charges that are created during switch actuation can dissipate through the substrate and be removed by the dc-grounded RF feed when in the "OFF" state. This method has been successfully documented in [6].

The reduction or elimination of bias lines is highly advantageous because they can significantly distort the radiation patterns and they can introduce additional unwanted resonances.

\section{RF MEMS Switch Design Procedure}

To change the actuation voltage of a MEMS switch, there are four parameters that can be changed.

1) Membrane material: Switch membranes are almost always made of metal. This is due to their pliable nature. Stiffer metals (that is, those with a high Young's Modulus $E$ ) will have a higher actuation voltage than those with a lower Young's Modulus.

2) Bridge thickness: The thicker the bridge, the stiffer the membrane. This gives a higher actuation voltage.

3) Bridge height: The higher the bridge, the larger the gap between the metal layers. This decreases the electrostatic force and increases the actuation voltage.

4) Membrane geometry: Springs can be designed into the shape of the membrane to lower the actuation voltage.

Of these parameters, only the fourth one does not require any fabrication changes. Making changes to a fabrication process can be a costly endeavor and may add additional variables. For example, it can be more challenging to precisely control the membrane height or thickness. For these reasons, we chose to alter the membrane geometry. By carefully controlling the spring constant $(\kappa)$ of the switch membrane, the actuation voltage can be tailored to a desired value.

\section{A. RF MEMS Switch Design and Simulation Results}

An accurate method for determining the actuation voltage for a given switch geometry was published in [13]. In this method, a switch is simulated using the static structural mechanics module from FEMLAB 3.0 [14]. FEMLAB, by Comsol, is 


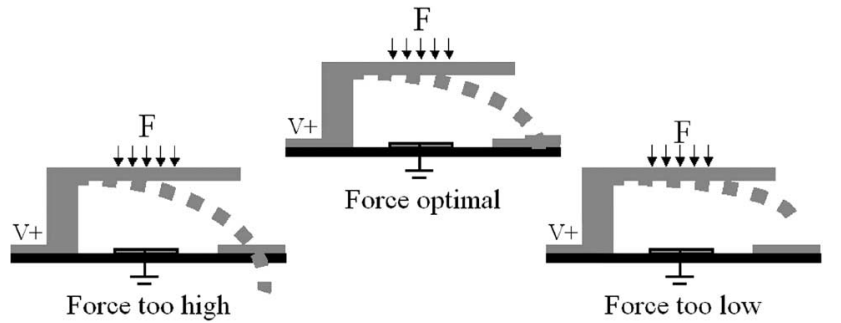

Fig. 3. Procedure for determining the optimal force needed to deflect the switch membrane. If the force is too high, the deflection is more than the membrane height. If the force is too low, the deflection does not reach the substrate. The optimal force is determined when the deflection matches the membrane height. The ground symbols denote the location of the floating ground.
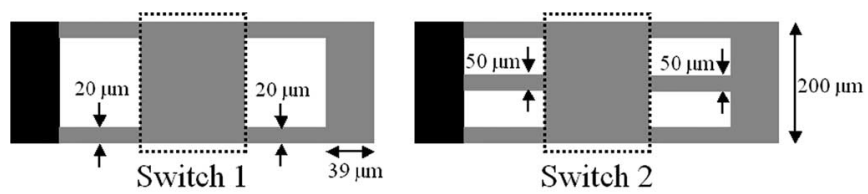

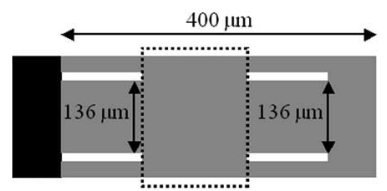

Switch 3

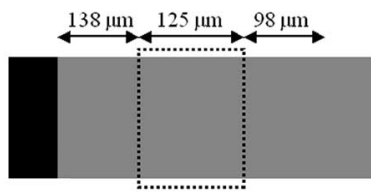

Switch 4
Fig. 4. Varieties of switch geometries are shown. The stationary posts are shown in black. The dotted areas show the electrostatic regions. The switches are listed from lowest actuation voltage (lowest spring constant) to the highest actuation voltage (highest spring constant). All dimensions are labeled.

a multiphysics simulator that uses the finite-element method. Any mechanical simulator that can perform a force-deflection analysis can use this method. Once the geometry and material specifications have been loaded into the software, a force can be applied to the beam over the electrostatic area, and the deflection can be determined. The force is changed until the deflection matches the desired bridge height. This procedure is demonstrated in Fig. 3.

The actuation voltage $V$ can then be calculated using

$$
V=\sqrt{\frac{2 g^{2} F}{\epsilon}}
$$

where $g$ is the gap (membrane height), $F$ is the force per area, and $\epsilon$ is the free-space permittivity. In [13], the measured voltage was within $5 \mathrm{~V}$ of the expected voltage.

For the mechanical simulations, it was assumed that an aluminum bridge with a thickness of $1.5 \mu \mathrm{m}$ was used that was suspended $5.0 \mu \mathrm{m}$ above the substrate. Aluminum has a Young's Modulus $(E)$ of $70 \mathrm{GPa}$, a Poisson's Ratio $(\nu)$ of 0.33 , and a density $(\rho)$ of $2700 \mathrm{~kg} / \mathrm{m}^{3}$. Single-supported (cantilever) ohmic switches were chosen although this technique could be used with other topologies. The switch geometries shown in Fig. 4 were loaded into FEMLAB with the mechanical and material properties stated before. These geometries were chosen because they have a wide variety of spring constants. They were also tuned to give a convenient ratio to the Switch 1 actuation voltage. That is, Switch 2 has an activation voltage that is 1.5 times higher than that of Switch 1 , and
TABLE I

Simulated Pull-Down Force and the Calculated Pull-Down Voltage From (1)

\begin{tabular}{|c|c|c|c|}
\hline Switch & Simulated Force (F) & Calculated Voltage (V) & $\frac{V_{\text {switch }}}{V_{\text {switch } 1}}$ \\
\hline 1 & $53.90 \mathrm{~N} / \mathrm{m}^{2}$ & $17.45 \mathrm{~V}$ & 1 \\
2 & $121.45 \mathrm{~N} / \mathrm{m}^{2}$ & $26.19 \mathrm{~V}$ & 1.5 \\
3 & $236.46 \mathrm{~N} / \mathrm{m}^{2}$ & $36.54 \mathrm{~V}$ & 2 \\
4 & $272.21 \mathrm{~N} / \mathrm{m}^{2}$ & $39.21 \mathrm{~V}$ & 2.25 \\
\hline
\end{tabular}
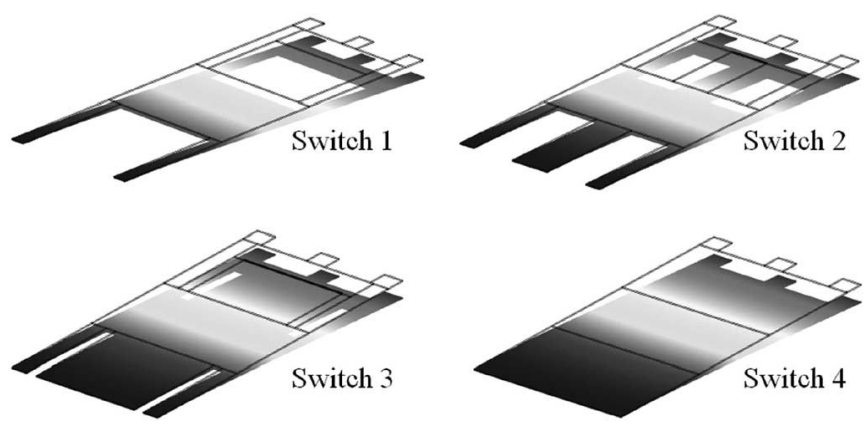

Fig. 5. Simulated deflections for the switch geometries from Fig. 4 are shown. The darkest areas represent the location of the posts where there is no deflection.

Switch 3 has an activation voltage that is two times higher than that of Switch 1, etc.

The simulated force that resulted in a $5-\mu \mathrm{m}$ deflection for each of the geometries is given in Table I. The deflection is shown in Fig. 5. These values were entered into (1) to calculate the pull-down voltage. These values are also given in Table I.

Careful considerations were made to ensure complete and symmetric actuation of the switch membranes. All the voltages presented in this paper are for the full pull-down state of the switch membrane. This was verified visually by increasing the pull-down voltage until the "ON"-state resistance and RF insertion loss values converged. Ohmic switches that are partially actuated will have a higher "ON"-state resistance and RF insertion loss than a fully actuated switch.

\section{B. RF MEMS Switch Measurement Results}

The switches shown in Figs. 4 and 5 were fabricated on LCP substrate. However, this technique would work for most substrates. The fabricated switches are shown in Fig. 6. They all have a measured resistance of $1.7 \Omega$ in the "ON" position and a measured capacitance of approximately $35 \mathrm{fF}$ in the "OFF" position. This low capacitance provides excellent isolation in the "OFF" position.

The minimum voltage was measured by starting at $0 \mathrm{~V}$ and increasing by $2 \mathrm{~V}$ every second. This increment was chosen because it is important to actuate the switch before substantial dielectric charging occurs. When the switch actuated, $S$ parameter measurements were taken. These results are shown in Figs. 7 and 8. The measured pull-down voltages agreed well with the expected values. These results are given in Table II.

\section{Antenna Design Procedure}

To date, Sierpinski gasket antennas have been fabricated on many different rigid substrates with low permittivity (such 


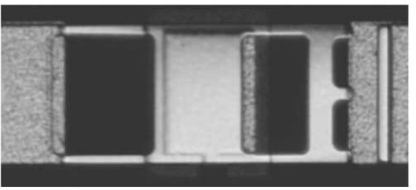

Switch 1

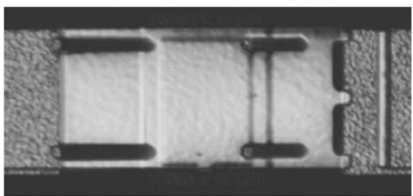

Switch 3

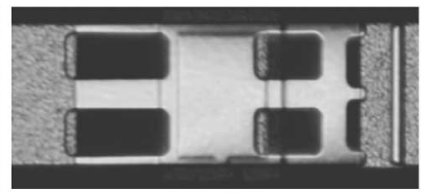

Switch 2

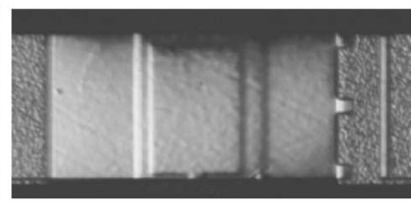

Switch 4
Fig. 6. Fabricated MEMS switches modeled after the designs shown in Figs. 4 and 5.

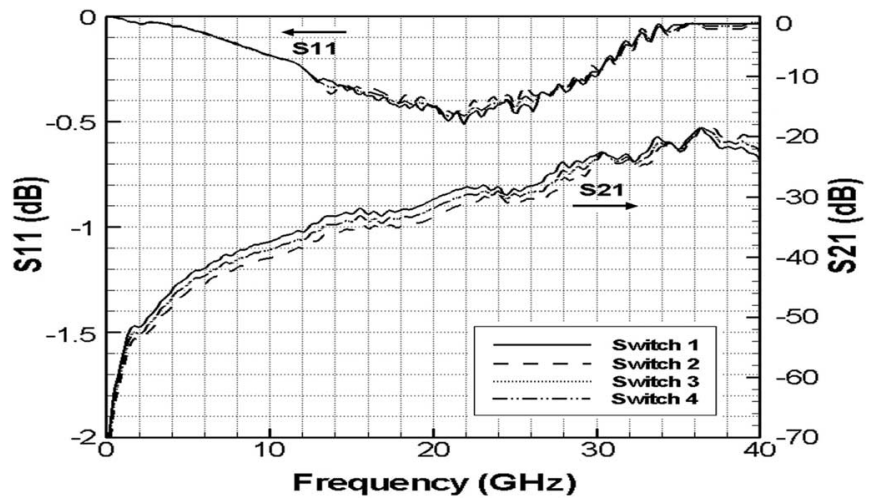

Fig. 7. $S$-parameter measurement results when the switch membrane is in the up position (not actuated).

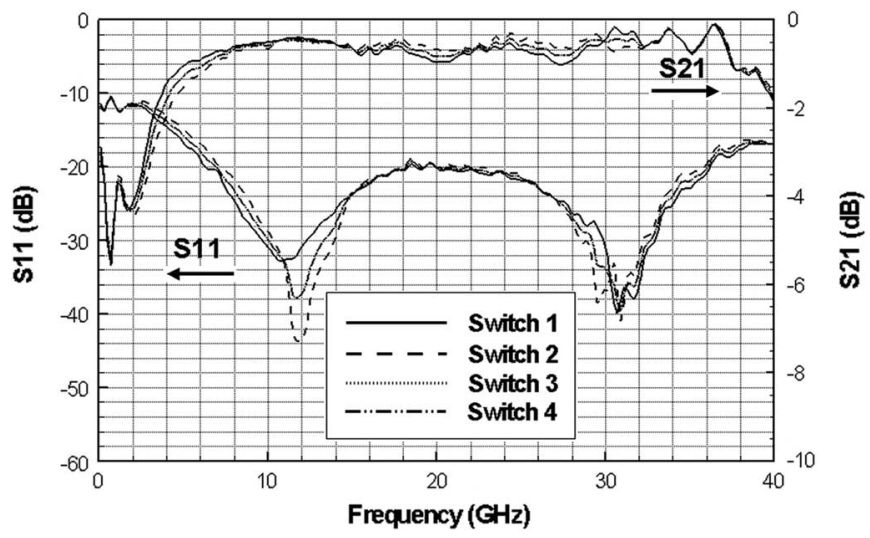

Fig. 8. $S$-parameter measurement results when the switch membrane is in the down position (actuated).

TABLE II

Comparison of Calculated and Measured Pull-Down Voltages. All Measured Voltages Are Within $2 \mathrm{~V}$ of the Actual Minimum Value Due to the 2-V/s InCREMENT

\begin{tabular}{|c|c|c|c|c|}
\hline Switch & Calculated V & Measured V & Difference & Percent Error \\
\hline 1 & $17.45 \mathrm{~V}$ & $18 \mathrm{~V}$ & $0.55 \mathrm{~V}$ & $3.15 \%$ \\
2 & $26.19 \mathrm{~V}$ & $28 \mathrm{~V}$ & $1.81 \mathrm{~V}$ & $6.91 \%$ \\
3 & $36.54 \mathrm{~V}$ & $38 \mathrm{~V}$ & $1.46 \mathrm{~V}$ & $4.00 \%$ \\
4 & $39.21 \mathrm{~V}$ & $42 \mathrm{~V}$ & $2.79 \mathrm{~V}$ & $7.12 \%$ \\
\hline
\end{tabular}

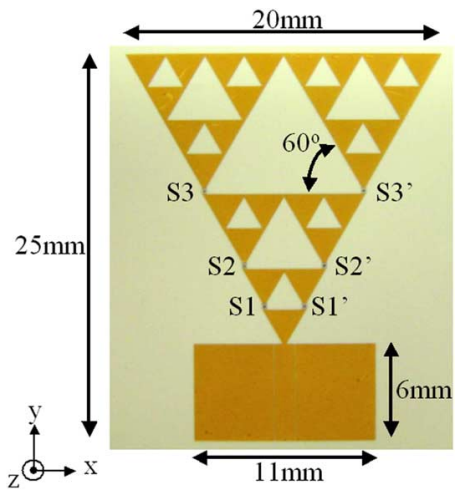

Fig. 9. Photograph of the fabricated Sierpinski antenna with MEMS switches shown. The design parameters are labeled on the plot.

as CuClad) and high permittivity (such as silicon). LCP was chosen as the substrate for its numerous advantages. LCP is a thin $(100 \mu \mathrm{m})$ flexible low-loss $(\tan \delta \approx 0.004)$ low-moistureabsorbing material with low permittivity $\left(\varepsilon_{r} \approx 3\right)$ [15]. Since the material is a polymer, there are additional packaging and cost advantages. All of these characteristics make it an ideal substrate for antennas, particularly at high frequencies.

With respect to the geometry, the antenna elements have a $60^{\circ}$ flare angle and maintain the resonant structure's selfsimilarity with a $\log$-periodicity of $\delta=2$. The antenna is fed through a 6-mm-long CPW transmission line with a $50-\mu \mathrm{m}$ gap, a 1.3-mm signal conductor width, and a $1.5-\mu \mathrm{m}$-thick aluminum layer. Switch geometries 1-3 from Figs. 4-6 were used to implement switches $\mathrm{S} 1, \mathrm{~S} 2$, and $\mathrm{S} 3$, respectively. A picture of the fabricated antenna is shown in Fig. 9. The overall size of the antenna, including the feed, is $20 \times 25 \mathrm{~mm}$.

The CPW feed was chosen to facilitate the measurement setup. This reconfigurable antenna operates at four different principle frequencies. For each of these frequencies, the antenna maintains its multiband performance.

The antenna was simulated using IE3D, ${ }^{1}$ a method-ofmoments electromagnetic solver. The simulated return loss is shown in Fig. 10. The switches were modeled in two ways. First, they were simplified to a $200 \times 400-\mu$ m gap in the "OFF" position and by a metal pad of the same size in the "ON" position. Those results were compared to a simulation that included the MEMS geometry in the "OFF" and "ON" positions. The difference in the results between the two simulations was minor, which indicates that the isolation provided by the MEMS was adequate.

It was verified that the antenna has a different first resonant frequency for each of the four states. Since the antenna is self-similar with a log-periodicity of two, each time the antenna transitions to the next state, the frequency should be halved. That is, the resonant frequency of state 2 should be half that of state 1 . The simulated $E$-plane copolarization ( $z y$-plane, $\varphi=90^{\circ}$ ) patterns for the four states are shown in Fig. 11. These patterns are as expected for a monopole antenna. The simulated radiation pattern for the $H$-plane copolarization

${ }^{1}$ IE3D is a trademark of Zeland Software 


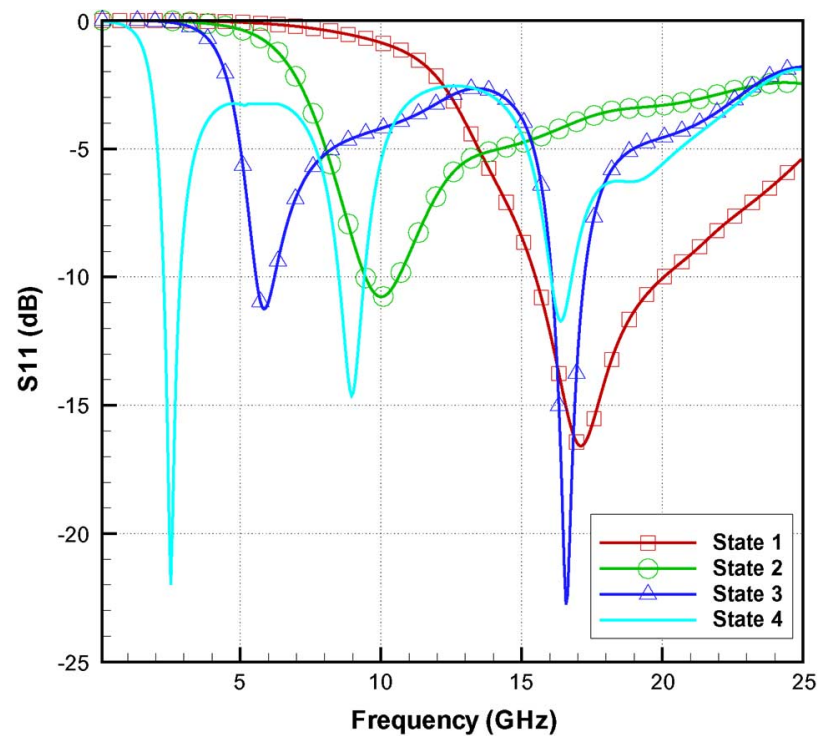

Fig. 10. Simulated return loss for all four states of the designed reconfigurable antenna.

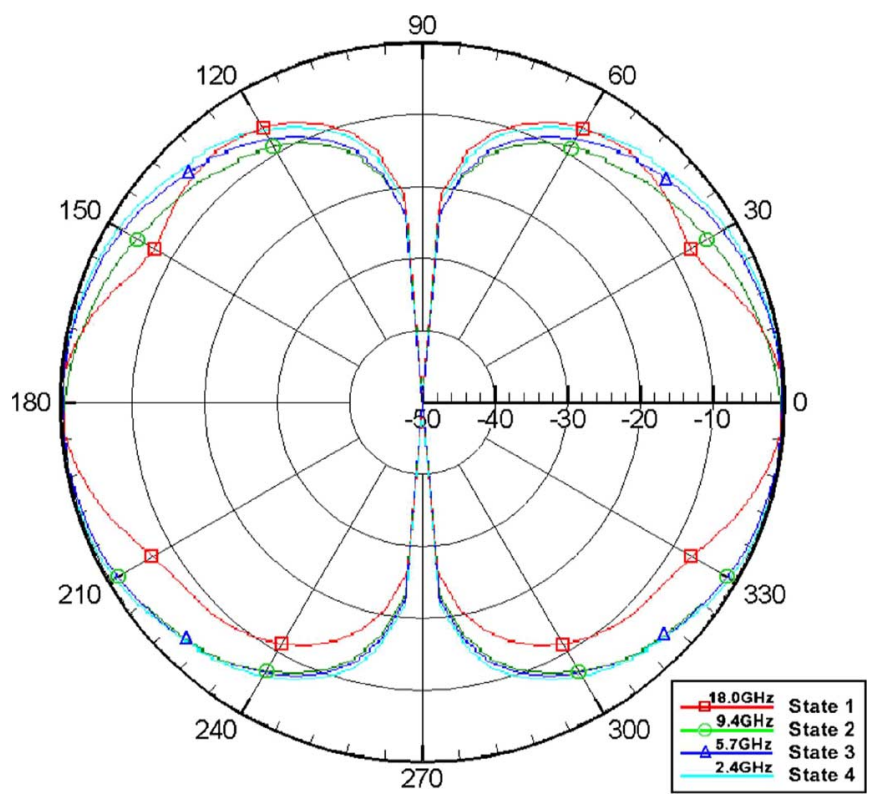

Fig. 11. Simulated radiation pattern for the $E$-total copolarization ( $z y$-plane, $\varphi=90^{\circ}$ ) plane for all four states of the designed reconfigurable antenna at the first resonant frequency. It is clear that the MEMS have a minimal effect on the antenna patterns, as it maintains its broadside characteristics.

( $x z$-plane, $\varphi=0^{\circ}$ ) is not presented for brevity since it shows an omnidirectional pattern in that plane.

\section{MEMS SWITCH INTEGRATION}

The placement of the RF MEMS switches was illustrated in Fig. 1 and shown in Fig. 9. In order to bias the ohmic switches for electrostatic actuation, the MEMS need to have an applied voltage. A metal pad beneath the switch should be present to attract the charged metal. The metal pad must be placed under a thin dielectric material (such as silicon nitride) to prevent direct metal bridge to metal pad contact. Otherwise, no charge will develop, and the switch will not actuate.

Traditionally, the actuation voltage is applied via a dc bias line. However, in order to prevent RF leakage into the dc path, careful attention needs to be given to the dc bias lines themselves. This can be implemented in different ways.

1) By using a quarter-wavelength transmission line connected to a quarter-wavelength open-circuit radial stub. Alternatively, a half-wavelength transmission line without a radial stub can be used with a reduced bandwidth. Each MEMS switch would require a different dc bias line and, for this antenna, that would require six lengthy metal lines being added. This would have a pronounced effect on the antenna performance. Therefore, this solution is not advisable.

2) High-resistance lines have been investigated to provide a wider bandwidth alternative [16]. Aluminum-doped zinc oxide is one example, which is used for biasing in [3]. Thin films of this kind are generally deposited using combustion chemical vapor deposition, which uses very high temperatures. This is not a problem for materials like silicon, but it is much higher than the melting point of the organic substrate $\left(\approx 315^{\circ} \mathrm{C}\right)$ used in this paper. At the moment, very high-resistivity materials that can be deposited at low temperature are not widely available but are under investigation [17].

The proposed alternative to these approaches is to eliminate the need for individual switch dc bias lines. Instead, the biasing is handled through the antenna structure itself. Here, the dc voltage and the RF signal are both applied to the antenna through the same signal conductor of the CPW feed line. The antenna reconfigurability is made possible by using MEMS switches of varying actuation voltages.

Like all RF MEMS devices, self-actuation of the switches can be an issue for the antenna. If the RF signal ever becomes large enough to actuate the switches, then the antenna will remain in state 4 . This will occur because these switches have an actuation time of approximately $40 \mu \mathrm{s}$. This is almost 100000 times slower than the period of the wavelength at $2.4 \mathrm{GHz}$ (the lowest operating frequency of the antenna). Effectively, all of the switches will remain in the "ON" position (state 4). This antenna should only be used at normal RF MEMS switch power levels (micro- to milliwatt range).

\section{ANTENNA AND MEMS FABRICATION}

Fabrication and MEMS integration was performed in six general steps. First, the LCP material was polished using an alumina slurry until the surface roughness was approximately $10 \mathrm{~nm}$. This roughness is comparable to that of a polished silicon wafer. Therefore, the original polymer roughness has no effect on the switch or the antenna performance. Second, the bottom seed layer was electron-beam deposited. Third, a silicon nitride layer was deposited using plasma-enhanced chemical vapor deposition, patterned, and etched using a reactive ion etch. Fourth, a sacrificial photoresist layer was patterned to define the switch height. Fifth, a $1.5-\mu \mathrm{m}$-thick 


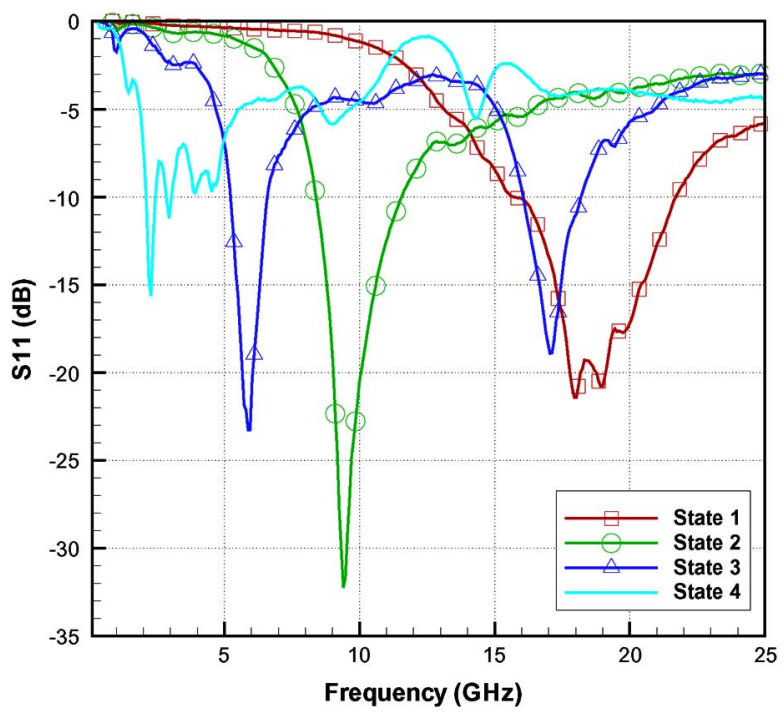

Fig. 12. Measured return loss for all four states of the designed reconfigurable antenna.

Ti-Al layer was electron-beam deposited and etched to define the switch membranes. Lastly, the switches were released by soaking in photoresist stripper and dried using $\mathrm{CO}_{2}$ at the super critical point.

\section{ReCONFigurable Sierpinski ANTENNA TESTING AND RESULTS}

The antenna reconfigurability was tested by varying the voltage and witnessing the antenna transition between the different states. The antenna was able to transition from the first to the last state and back again without a change in the performance. This procedure was repeated many times without problems. This demonstrates that the floating ground is sufficient.

The return-loss measurements were taken with an Agilent $8510 \mathrm{C}$ vector network analyzer using $850-\mu \mathrm{m}$ pitch GSG RF probes. Pattern measurements were taken using an Agilent 8530 vector network analyzer with the antenna inside an anechoic chamber. End-launch gold SMA connectors were hand soldered onto the antenna for pattern measurements. These connectors have a maximum operating frequency of $18 \mathrm{GHz}$, which coincides with the highest principle frequency of the antenna when no voltage is applied.

The return-loss-measurement results are shown in Fig. 12. The resonant frequencies roughly halve as the antenna increases in size. These measurement results are summarized in Table III and agree well with the simulated values shown in Fig. 10. As a proof of concept, the focus was given to the first resonances only, and thus, higher order modes were not studied in great detail. Higher order modes of the Sierpinski gasket antennas have been studied in [11] and [12]. However, all of the first resonance frequencies were correctly simulated. Almost all of the higher order resonances were measured within $5 \%$ of the simulated values.

The state 4 measurement results exhibit the most variance from the simulated values because of the size of the antenna. This accounts for the second $(9.0 \mathrm{GHz})$ and third $(16.4 \mathrm{GHz})$
TABLE III

Tabulated Antenna Simulation (and Measurement) Results for All Four States. The ACtuation Voltage and First Three Resonances (In Gigahertz) Are Given. The MaXimum PERCENT ERRor BETWEen SimUlated AND MEASURED VALUES IS NOTED

\begin{tabular}{|c|c|c|c|c|}
\hline State & Voltage & $f_{1}(\mathrm{GHz})$ & $f_{2}(\mathrm{GHz})$ & $f_{3}(\mathrm{GHz})$ \\
\hline 1 & $0 \mathrm{~V}$ & $18.0(18.0)$ & $>30(>30)$ & $>30(>30)$ \\
\hline 2 & $18 \mathrm{~V}$ & $9.4(9.4)$ & $>30(>30)$ & $>30(>30)$ \\
\hline 3 & $28 \mathrm{~V}$ & $5.7(5.7)$ & $16.7(17.5)$ & $>30(>30)$ \\
\hline 4 & $38 \mathrm{~V}$ & $2.4(2.4)$ & $9.0(9.0)$ & $16.4(14.3)$ \\
\hline \multicolumn{2}{|c|}{ Maximum error } & $(0 \%)$ & $(-4.8 \%)$ & $(14.3 \%)$ \\
\hline
\end{tabular}

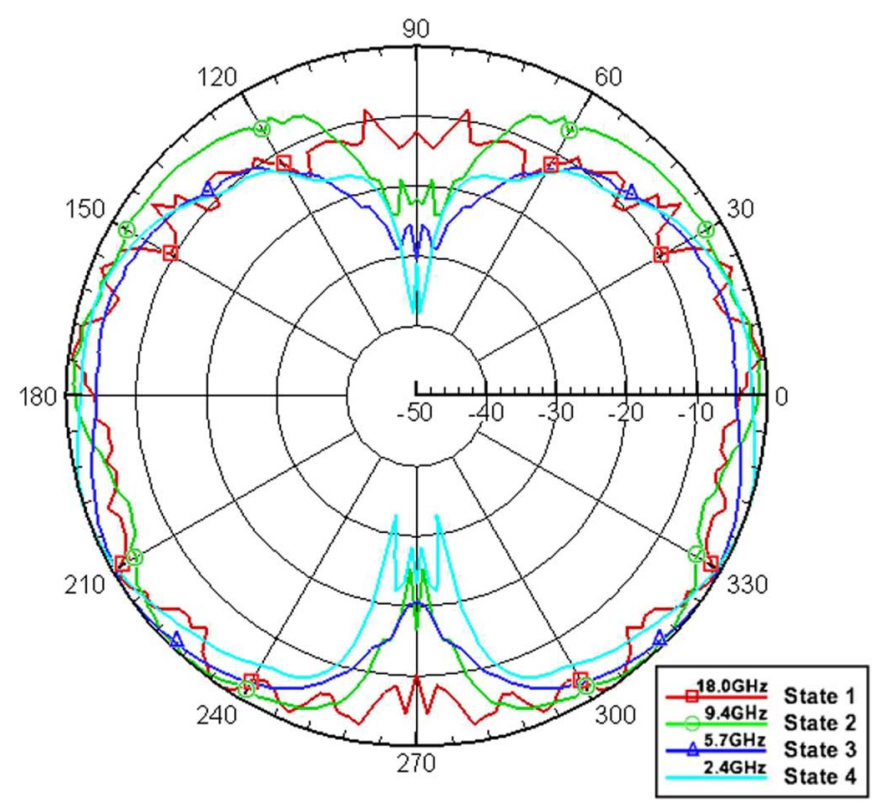

Fig. 13. Measured radiation pattern for the $E$-theta copolarization ( $z y$-plane, $\varphi=90^{\circ}$ ) plane for all four states of the designed reconfigurable antenna at the first resonant frequency. Broadside radiation with similar patterns at each frequency is achieved.

resonances that are visible in the measured results but are not as pronounced as in the simulations.

The measured normalized patterns for the $E$-plane copolarization $\left(z y\right.$-plane, $\left.\varphi=90^{\circ}\right)$ are shown in Fig. 13. Some ripple can be noticed in state 1 of the antenna due to mismatch from the coaxial SMA connector. The measured patterns agree well with the simulated ones shown in Fig. 11. For clarity, these plots are not superimposed. The measured radiation pattern for the $H$-plane copolarization ( $x z$-plane, $\varphi=0^{\circ}$ ) is not presented as before since it shows an omnidirectional pattern in that plane.

\section{CONCLUSION}

This paper has presented the possibility of adding an additional level of reconfigurability to a device or system by simply integrating RF MEMS switches with different geometries. A sequentially reconfigurable RF MEMS multiband antenna was designed, fabricated, and tested on a flexible organic substrate for the first time. The purpose of this paper was not only to 
illustrate a method of biasing MEMS reconfigurable antennas without the need for dc bias lines but also to illustrate how the antenna performance can be enhanced by increasing the number of resonant frequencies. The final device does not have any additional lines to bias the switches, while the antenna exhibited four principle resonant frequencies with good radiation characteristics. By using MEMS switches, the losses are kept to a minimum. Three different switch geometries were integrated into a Sierpinski antenna with different actuation voltages. The simulated and measured responses agree well. This technology can be applied to many other devices, including tuners, tunable filters, other antenna geometries, or signal splitters.

\section{REFERENCES}

[1] C. D. Nordquist et al., "An X-band to Ku-band RF MEMS switched coplanar strip filter," IEEE Microw. Wireless Compon. Lett., vol. 14, no. 9, pp. 425-427, Sep. 2004.

[2] R. Lempkowski, K. Lian, M. Eliacin, and P. Kulkarni, "A PWBbased MEMS switched filter bank using lumped element embedded passives," in Proc. 31st Annu. Conf. IEEE Ind. Electron. Soc., Nov. 2005, pp. 2331-2334.

[3] D. E. Anagnostou et al., "Design, fabrication, and measurements of an RF-MEMS-based self-similar reconfigurable antenna," IEEE Trans. Antennas Propag., vol. 54, no. 2, pt. 1, pp. 422-432, Feb. 2006.

[4] B. Cetiner, J. Qian, H. Chang, M. Bachman, G. Li, and F. DeFlaviis, "Monolithic integration of RF MEMS switches with a diversity antenna on PCB substrate," IEEE Trans. Microw. Theory Tech., vol. 51, no. 1, pp. 332-334, Jan. 2003.

[5] R. Jackson and R. Ramadoss, "A MEMS-based electrostatically tunable circular microstrip patch antenna," J. Micromech. Microeng., vol. 17, no. 1, pp. 1-8, Jan. 2007.

[6] N. Kingsley and J. Papapolymerou, "Organic 'wafer-scale' packaged miniature four-bit RF MEMS phase shifter," IEEE Trans. Microw. Theory Tech., vol. 54, no. 3, pp. 1229-1236, Mar. 2006.

[7] C. P. Baliarda, J. Romeu, and A. Cardama, "The Koch monopole: A small fractal antenna," IEEE Trans. Antennas Propag., vol. 48, no. 11, pp. 1773-1781, Nov. 2000.

[8] S. R. Best, "On the resonant properties of the Koch fractal and other bent wire monopole antennas," IEEE Antennas Wireless Propag. Lett., vol. 1, no. 1, pp. 74-76, 2002.

[9] N. Cohen and R. Hohlfeld, "Fractal loops and the small loop approximation," Commun. Q., pp. 77-81, Winter 1996.

[10] D. Werner and S. Ganguly, "An overview of fractal antenna engineering research," IEEE Antennas Propag. Mag., vol. 45, no. 1, pp. 38-57, Feb. 2003

[11] C. Puente Baliarda, J. Romeu, R. Pous, and A. Cardama, "On the behavior of the Sierpinski multiband fractal antenna," IEEE Trans. Antennas Propag., vol. 46, no. 4, pp. 517-524, Apr. 1998.

[12] J. Anguera, C. Puente, C. Borja, and J. Soler, "Fractal-shaped antennas: A review," in Wiley Encyclopedia of RF and Microwave Engineering, vol. 2, K. Chang, Ed. New York: Wiley, 2005, pp. 1620-1635.

[13] N. Kingsley, G. Wang, and J. Papapolymerou, "Comparative study of analytical and simulated doubly-supported RF MEMS switches for mechanical and electrical performance," Appl. Comput. Electromagn. Soc. J., vol. 21, no. 1, pp. 9-15, Mar. 2006.

[14] Comsol Inc., FEMLAB Multiphysics Modeling, Nov. 2004. [Online]. Available: http://www.comsol.com/products/femlab

[15] D. Thompson, O. Tantot, H. Jallageas, G. Ponchak, M. Tentzeris, and J. Papapolymerou, "Characterization of liquid crystal polymer (LCP) material and transmission lines on LCP substrates from 30 to $110 \mathrm{GHz}$," IEEE Trans. Microw. Theory Tech., vol. 52, no. 4, pp. 1343-1352, Apr. 2004.

[16] G. Du, Y. Zhang, Y. Ma, X. Yang, B. Zhao, and B. Liu, "ZnO thin films grown by plasma-assisted metal-organic vapor phase epitaxy," in Proc. 6th Chin. Symp. Optoelectronics, Sep. 2003, pp. 292-296.

[17] S. Horst, S. Bhattacharya, S. Johnson, E. Tentzeris, and J. Papapolymerou, "Modeling and characterization of thin film broadband resistors on LCP for RF applications," in Proc. 56th Electron. Compon. Technol. Conf., Jun. 2006, pp. 1751-1755.

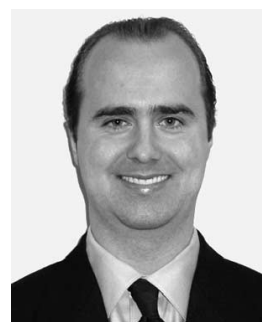

Nickolas Kingsley (S'02-M'07) received the B.S., M.S., and Ph.D. degrees in electrical engineering from the Georgia Institute of Technology (Georgia Tech), Atlanta, in 2002, 2004, and 2007, respectively.

During his thesis work, he investigated methods for packaging RF microelectromechanical systems switches in flexible organic packages and tested the methods for reliability. In June 2007, he joined the Auriga Measurement Systems team in Lowell, MA, as a Principal Engineer. He will be developing high-performance amplifier systems. His research interests have focused on developing miniature multilayer system-on-package RF front ends using liquid crystal polymer substrate. He has published one book chapter and over a dozen publications and has submitted four invention disclosures.

Dr. Kingsley was a member of the Georgia Electronic Design Center under the direction of Prof. J. Papapolymerou until May 2007. He is a member of the IEEE Antennas and Propagation Society, IEEE Microwave Theory and Techniques Society, and the Order of the Engineer. While at Georgia Tech, he won numerous awards. He is the recipient of the 2002 President's Undergraduate Research Award. He won three poster competitions at university, college, and school levels. He received the Trainer of the Year distinction from the Microelectronics Research Center clean room in 2005 and 2006. As a coop student with Compaq Computer Corporation, he won the 2001 Armada Award for excellence. He will serve as a Technical Program Committee member for the 2008 International Microwave Symposium Conference in Atlanta, GA.

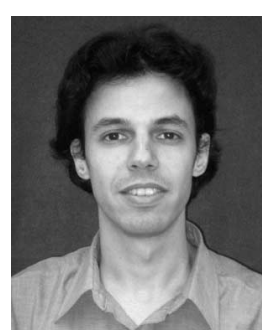

Dimitrios E. Anagnostou (S'98-M'05) was born in Athens, Greece, in November 1975. He received the Diploma degree (M.Sc. equivalent) in electrical and computer engineering from Democritus University of Thrace, Xanthi, Greece, in 2000, and the M.Sc. and $\mathrm{Ph} . \mathrm{D}$. degrees in electrical engineering from the University of New Mexico, Albuquerque, in 2002 and 2005, respectively.

From August 2005 to December 2006, he was a Postdoctoral Fellow with the School of Electrical and Computer Engineering, Georgia Institute of Technology, Atlanta. Since 2007, he has been with the faculty of Electrical and Computer Engineering Department, South Dakota School of Mines and Technology, Rapid City, as an Assistant Professor. His current research interests include the development, characterization, and integration of reconfigurable low-cost flexible antennas and RF front ends using direct write deposition processes, miniaturized antenna designs, microwave packaging, RF microelectromechanical systems, and neural networks. He has authored or coauthored more than 25 papers in published peer-reviewed journals and international conference proceedings.

Dr. Anagnostou has been awarded with three research and travel grants from 2003 to 2005 and has filed two invention disclosures on reconfigurable and ultrawideband antennas. He is serving as a Reviewer for the IEEE Transactions on Antennas and Propagation, the IEEE Transactions on Microwave Theory and Techniques, and other international publications. In 2006 and 2007, he was invited to act as a Session Chair for the IEEE International Symposia on Antennas and Propagation. He is a member of Eta Kappa Nu and the Technical Chamber of Greece. 


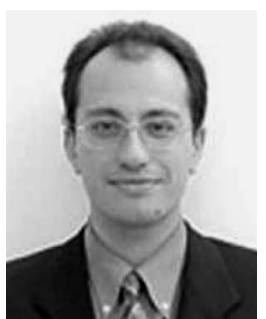

Manos Tentzeris (SM'07) received the Diploma degree in electrical and computer engineering (magna cum laude) from the National Technical University of Athens, Athens, Greece, and the M.S. and Ph.D. degrees in electrical engineering and computer science from the University of Michigan, Ann Arbor.

He was a Visiting Professor with the Technical University of Munich, Germany, for the summer of 2002 , where he introduced a course in the area of high-frequency packaging. He is the Georgia Electronic Design Center Associate Director for RF identifications (RFIDs)/sensors research, and he has been the Georgia Tech National Science Foundation (NSF) Packaging Research Center Associate Director for RF research and the RF Alliance Leader from 2003 to 2006. He is also the Leader of the RFID Research Group of the Georgia Electronic Design Center of the State of Georgia. He is currently an Associate Professor with the School of Electrical and Computer Engineering, Georgia Institute of Technology, Atlanta. $\mathrm{He}$ has helped develop academic programs in highly integrated/multilayer packaging for RF and wireless applications using ceramic and organic flexible materials, paper-based RFIDs and sensors, microwave microelectromechanical systems, system-on-package-integrated (ultrawideband, multiband, conformal) antennas, and adaptive numerical electromagnetics (finite-difference time-domain, MultiResolution Algorithms). He is the Head of the ATHENA Research Group of 20 researchers. He has published more than 250 papers in refereed journals and conference proceedings, two books, and ten book chapters. He has given more than 50 invited talks in the area of high-frequency packaging to various universities and companies in Europe, Asia, and America.

Dr. Tentzeris was the recipient of the 2007 IEEE Antennas and Propagation Society (APS) Symposium Best Student Paper Award, the 2007 IEEE Instrumentation and Measurement Society (IMS) Symposium ThirdPlace Best Student Paper Award, the 2006 IEEE Microwave Theory AND Techniques (MTT) Outstanding Young Engineer Award, the 2006 Asian-Pacific Microwave Conference Award, the 2004 IEEE TRANS ACTIONS on Advanced Packaging Commendable Paper Award, the 2003 NASA Godfrey "Art" Anzic Collaborative Distinguished Publication Award, the 2003 IBC International Educator of the Year Award, the 2003 IEEE Components, Packaging, and Manufacturing Technology Society (CPMT) Outstanding Young Engineer Award, the 2002 International Conference on Microwave and Millimeter-Wave Technology Best Paper Award, Beijing, China, the 2002 Georgia Tech-ECE Outstanding Junior Faculty Award, the 2001 ACES Conference Best Paper Award, the 2000 NSF CAREER Award, and the 1997 Best Paper Award of the International Hybrid Microelectronics and Packaging Society. He was also the 1999 Technical Program Cochair of the 54th ARFTG Conference, Atlanta, GA, and the Chair of the 2005 IEEE Computational Electromagnetics in Time Domain Workshop, and he is the Vice Chair of the RF Technical Committee (TC16) of the IEEE CPMT Society. He has organized various sessions and workshops on $\mathrm{RF} /$ wireless packaging and integration, RFIDs, numerical techniques/wavelets for the IEEE Electronics Components and Technology Conference, IMS, Vehicular Technology Conference, and APS Symposia in all of which he is a member of the Technical Program Committee in the area of "Components and RF." He will be the Transaction Processing Performance Council Chair for the IEEE IMS 2008 Symposium. He is an Associate Editor of the IEEE TRANSACTIONS ON ADVANCED PACKAGING. $\mathrm{He}$ is a member of Union Radio-Scientifique Internationale-Commission D, a member of MTT-15 committee, an Associate Member of EuMA, and a member of the Technical Chamber of Greece.

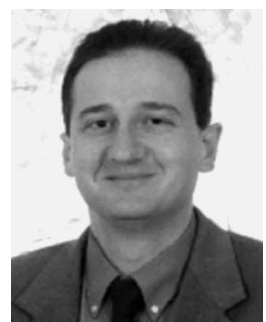

John Papapolymerou (SM'04) received the B.S.E.E. degree from the National Technical University of Athens, Athens, Greece, in 1993, and the M.S.E.E. and Ph.D. degrees from the University of Michigan, Ann Arbor, in 1994 and 1999, respectively.

From 1999 to 2001, he was a Faculty Member with the Department of Electrical and Computer Engineering, University of Arizona, Tucson, and during the summers of 2000 and 2003, he was a Visiting Professor at the University of Limoges, France. From 2001 to 2005, he was an Assistant Professor with the School of Electrical and Computer Engineering, Georgia Institute of Technology, Atlanta, where he is currently an Associate Professor. His research interests include the implementation of micromachining techniques and microelectromechanical systems devices in microwave, millimeter-wave and terahertz circuits, and the development of both passive and active planar circuits on semiconductor $(\mathrm{Si} / \mathrm{SiGe}$, $\mathrm{GaAs}$ ), and organic substrates (liquid crystal polymer, low-temperature cofired ceramic) for system-on-a-chip/system-on-a-package RF front ends. He has authored or coauthored over 150 publications in peer-reviewed journals and conference proceedings.

Dr. Papapolymerou received the 2004 Army Research Office Young Investigator Award, the 2002 National Science Foundation CAREER Award, the best paper award at the Third IEEE International Conference on Microwave and Millimeter-Wave Techmology (ICMMT2002), Beijing, China, and the 1997 Outstanding Graduate Student Instructional Assistant Award presented by the American Society for Engineering Education, University of Michigan Chapter. In 2004, he was the Chair of the IEEE Microwave Theory and Techniques Society/AP Atlanta Chapter. He currently serves as the Vice Chair for Commission D of the U.S. National Committee of the Union Radio-Scientifique Internationale and as an Associate Editor for the IEEE TRANSACTIONS ON Antennas and Propagation and IEEE Microwave and Wireless COMPONENT LETTERS. 